\title{
Candida albicans isoladas da cavidade bucal de crianças com síndrome de Down: ocorrência e inibição do crescimento por Streptomyces sp
}

\author{
Candida albicans isolated from buccal cavity of children with Down's syndrome: \\ occurrence and growth inhibition by Streptomyces sp
}

\author{
José Daniel Gonçalves Vieira ${ }^{1}$, Evandro Leão Ribeiro ${ }^{1}$, Cerise de Castro Campos ${ }^{2}$, \\ Fabiana Cristina Pimenta ${ }^{1}$, Orlando Ayrton de Toledo ${ }^{2}$, Gustavo Morais Nagato ${ }^{3}$, \\ Niwmar Alves de Souza ${ }^{3}$, Wesley Magno Ferreira ${ }^{4}$, Cléver Gomes Cardoso ${ }^{5}$, \\ Sueli Meira da Silva Dias ${ }^{6}$, César Aparício de Araújo Júnior ${ }^{7}$, Daniel Teles Zatta \\ e Juliana de Sousa dos Santos ${ }^{7}$
}

\begin{abstract}
RESUM0
Comparação entre a presença de leveduras de Candida na cavidade bucal de crianças sem e com síndrome de Down mostrou-se estatisticamente significante no caso de crianças afetadas por esta cromossomopatia, tornando-as mais predispostas à candidíase bucal, provavelmente favorecida pelas alterações anátomo-fisiológicas da boca em decorrência da trissomia do cromossomo 21. Recidivas constantes de candidíase bucal em crianças portadoras desta alteração cromossômica levou a busca de prováveis alternativas terapêuticas. Visando determinar a atividade antifúngica de Streptomyces sp isolados de diferentes solos brasileiros, 5 cepas foram testadas frente a Candida albicans, oriundas da cavidade bucal de crianças com síndrome de Down. Observou-se que os isolados apresentaram uma diversidade de tamanho dos halos ( 9-31 mm de diâmetro) de inibição de crescimento das leveduras, sugerindo uma possível utilização em terapêutica antifúngica.
\end{abstract}

Palavras-chaves: Candida albicans. Síndrome de Down. Streptomyces sp.

\begin{abstract}
Comparison of the presence of Candida yeasts in the buccal cavity of children without and with Down's syndrome showed a statistically significant difference in the case of children that were affected by this chromosomopathy, rendering them more predisposed to buccal candidiasis, probably due to anatomicophysiologic alterations of the mouth resulting from trisomy of chromosome 21. Constant recurrence of buccal candidiasis in children with this chromosomal alteration lead to the search for a possible therapeutic alternative. Seeking to determine the antifungal activity of Streptomyces sp isolated from various Brazilian soils, 5 strains have been tested for Candida albicans isolated from the buccal cavity of children with Down's syndrome. It was observed that the isolate presented a diversity in the size of the halos (9-31 mm in diameter) of growth inhibition of the yeasts, suggesting a possible use as a therapeutic antifungal.
\end{abstract}

Key-words: Candida albicans. Down's syndrome. Streptomyces sp.

1. Departamento de Microbiologia do Instituto de Patologia Tropical e Saúde Pública da Universidade Federal de Goiás, Goiânia, G0. 2. Departamento de Odontologia da Faculdade de Ciências da Saúde da Universidade de Brasília, Brasília, DF. 3. Cirurgiões-dentistas da Universidade Federal de Goiás, Goiânia, GO. 4. Especialista em Farmácia Hospitalar e Assistencial da Universidade Federal de Goiás, Goiânia, GO. 5. Doutorando em Patologia Molecular da Universidade de Brasília, Brasília, DF. 6. Mestranda em Medicina Tropical do Instituto de Patologia Tropical e Saúde Pública da Universidade Federal de Goiás, Goiânia, G0. 7. Farmacêuticos da Universidade Federal de Goiás, Goiânia, GO.

Endereço para correspondência: Dr José Daniel G. Vieira. R. Delenda Resende de Melo s/n. Setor Universitário 74505-220 Goiânia, G0.

e-mail: jvieira@ iptsp.ufg.br, evandro0@ terra.com.br, wesleymf@ terra.com.br, cardosocg@ unb.br

Recebido para publicação em 23/3/2004

Aceito em 6/6/2005 
Asleveduras do gênero Candida são microrganismosintegrantes da microbiota bucal do homem desde 0 nascimento ${ }^{2}$. Esta condição microbiológica propicia comumente uma relação de equilíbrio entre parasita-hospedeiro, diante da manutenção da integridade das barreiras teciduais, relação harmônica da microbiota autóctone e funcionamento adequado do sistema imunológico humano, havendo em contrapartida por parte do fungo leveduriforme, permanência equilibrada da capacidade de aderência e da produção de enzimas e toxinas. Alterações físicas, químicas, iatrogênicas e mecânicas, que se processem na cavidade bucal, como a mastigação, possam entre diversos fatores descritos favorecer a ruptura do equilíbrio estabelecido entre 0 fungo e 0 hospedeiro fazendo com que as infecções por Candida sejam de origem geralmente endógena ${ }^{23}$.

Nas crianças com síndrome de Down, além das alterações anátomo-fisiológicas bucais, macroglossia, estagnação salivar decorrente de incompetência muscular da boca, dificuldade motora, constantes doenças respiratórias e comprometimento simultâneo da resposta imunológica inata e adquirida fazem com que estes fatores adicionais, astornem mais suscetiveis a processos infecciosos, inclusive fúngicos, onde as espécies de Candida são os agentes etiológicos mais preponderantes ${ }^{4} 7171820$. Este sítio bucal altamente povoado por cepas de Candida faz também com que as crianças detentoras desta alteração cromossômica possuam o biofilme dentário com uma interferência fúngica maior, fazendo com que haja uma ação direta ou coadjuvantena ocorrência da cárie dentária, gengivite e periodontite, quando os estreptococos do grupo mutans atuam como agentes principais $^{3121519}$. Candidíase pseudomembranosa é 0 quadro clínico fúngico mais detectável em crianças com esta cromossomopatia ${ }^{34710}$. Todavia, cerca de 20 a 55\% da população assindrômica podem apresentar isolados de Candida como leveduras participantes da mucosa bucal do homem ${ }^{18} 1925$.

Drogas azólicas e antibióticos poliênicos constituem os recursos terapêuticos mais comumente empregados em candidíase bucal. Entretanto, constantes recidivas de manifestações de Candida na boca de crianças, inclusive nas portadoras de síndrome de Down, favorecem a busca de novas drogas ${ }^{11}$.

Os antibióticos são exemplos de metabólitos secundários produzidos por certos microrganismos que possuem a capacidade de inibir o crescimento de seres microbiológicos, quando utilizados em baixas concentrações ${ }^{15}$. Do grande número de antibióticos conhecidos de origem microbiana, somente 123 são produzidos atualmente por fermentação. Além disto, mais de 50 antibióticos são produzidos como compostos semi-sintéticos e 3 antibióticos são sinteticamente produzidos ${ }^{6212}$. Entre as bactérias, a maior variedade em estruturas e número de antibióticos é encontrada nos actinomicetos, especialmente no gênero Streptomyces ${ }^{22}$.

0 isolamento de novos actinomicetos, principalmente Streptomyces, com atividade antifúngica tem sido apontado com grande entusiasmo no descobrimento de novos agentes antifúngicos com potencial de emprego terapêutico. Este isolamento está intimamente associado a técnicas clássicas para isolamento e seleção ( técnica de diluição e inoculação em ágar com posterior utilização de fungos como alvos de seleção) ${ }^{13}$. Este trabalho teve por objetivo verificar a ocorrência e determinar a atividade antifúngica de Streptomyces sp, isolados dos solos da Mata Atlântica do Estado do Espírito Santo (ES) e do Cerrado brasileiro frente às cepas de Candida oriundas da cavidade bucal de crianças com síndrome de Down.

\section{MATERIAL E MÉTODOS}

Isolamento e identificação. Setenta amostras de secreção salivar foram coletadas com swabs esterilizados da mucosa jugal de crianças, sendo 35 amostras de crianças portadoras de síndrome de Down (grupo-teste) e as demais crianças desprovidas desta síndrome ( grupo controle). Todas as crianças possuíam faixa etária de 0 a 10 anos, apresentavam mucosa bucal íntegra, não faziam uso de nenhuma medicação e foram atendidas na Clínica de Odontopediatria da Faculdade de Odontologia da Universidade Federal de Goiás ( FO/UFG) em Goiânia-G0, Brasil. Após a homogeneização, as amostras de saliva foram semeadas em duplicata em meio ágar Sabouraud dextrose com cloranfenicol e mantidas a temperatura ambiente por 15 dias no Laboratório de Micologia do Instituto de Patologia Tropical e Saúde Pública ( IPTSP/UFG) . As colônias leveduriformes brancoamareladas desenvolvidas foram identificadas mediante pesquisa de tubo germinativo em soro fetal bovino, formação de clamidósporos em ágar corn-meal acrescido de tween $80 \mathrm{e}$ realização de testes de assimilação e fermentação de carbon ${ }^{810}$. Este estudo foi previamente aprovado pelo Comitê de Ética em Pesquisa Médica Humana e Animal do Hospital das Clínicas da Universidade Federal de Goiás, e os pais e/ou responsáveis pelas crianças forneceram consentimento aos pesquisadores.

Estatística. Foi realizada aplicando 0 teste de $\chi^{2}$ (Quiquadrado) para correlacionar os índices de carreamento de leveduras de Candida entre as cavidades bucais de crianças com e sem síndrome de Down e os valores obtidos foram considerados estatisticamente significantes quando $\mathrm{p}<0,05$.

Cepas de Candida utilizadas na análise antifúngica. Foram escolhidas aleatoriamente 8 cepas de Candida isoladas e identificadas dentre as provenientes da mucosa bucal de crianças com síndrome de Down. Foi utilizado como referência a cepa de C. albicans CBS562, previamente cedida pela micoteca do Instituto de Ciências Biomédicas da Universidade de São Paulo (ICB/USP) Brasil.

Isolados de Streptomyces sp utilizados. Foram utilizados 5 isolados de Streptomyces sp, provenientes dos solos da Mata Atlântica (ES) (TIJA 2, TIJA 12 e TILA 1) e do Cerrado brasileiro (ADU 1.3 e ADU 2.1). Estes isolados fazem parte da bacterioteca do Laboratório de Microbiologia Ambiental e Biotecnologia (IPTSP/UFG) ${ }^{24}$.

Detecção de atividade antifúngica. Esporos dos Streptomyces sp foram cultivados em meio ágar ISP-2 (constituído de g/L: glucose 4; extrato de levedura 4; extrato de malte 10; ágar-ágar $20, \mathrm{pH} 7,3$ ) a $30^{\circ} \mathrm{C}$ por 15 dias até esporulação para a produção das possíveis substâncias antifúngicas. Após este tempo, plugs de $7 \mathrm{~mm}$ de diâmetro foram cortados e inoculados em placas de Petri, contendo o meio de ágar Sabouraud dextrose, onde as cepas-teste de C. albicans e 
também da cepa indicadora C. albicans CBS 562 foram previamente semeadas com auxílio de swab. Estas placas foram incubadas a $30^{\circ} \mathrm{C}$ por 2 dias até 0 desenvolvimento de halos de inibição de crescimento. Os diâmetros ( $\mathrm{mm}$ ) destes halos de inibição foram determinados segundo Vieira ${ }^{24}$.

\section{RESULTAD0S}

Das 70 amostras de secreção salivar coletadas das crianças detectou-se a ocorrência de Candida em 36 ( 51,4\%) amostras, sendo todas pertencentes à espécie albicans. A diferença entre a detecção de leveduras de Candida carreadas entre as mucosas bucais de crianças com e sem síndrome de Down mostrou significância estatística $(p<0,05)$ (Tabela 1).
Tabela 1 - Ocorrência de cepas de Candida albicans isoladas da mucosa jugal de crianças atendidas na Clínica de Odontopediatria da FO/UFG.

\begin{tabular}{lcccccc}
\hline Cepas & \multicolumn{2}{c}{$\begin{array}{c}\text { Crianças com } \\
\text { síndrome de Down }\end{array}$} & & \multicolumn{2}{c}{$\begin{array}{c}\text { Crianças sem } \\
\text { síndrome de Down* }\end{array}$} \\
\cline { 2 - 3 } \cline { 5 - 6 } & $\mathrm{n}^{\mathbf{0}}$ & $\%$ & & $\mathrm{n}^{0}$ & $\%$ \\
\hline Candida albicans & 30 & 85,7 & & 6 & 17,1 \\
\hline Total & \multicolumn{2}{c}{35} & & \multicolumn{2}{c}{35} \\
\hline
\end{tabular}

*Relação estatística entre os grupos CCSD e CSSD $\left(\chi^{2}=32,9\right.$; $(\mathrm{p}<0,05)$

Atividade antifúngica. Observou-se que todas as cepas de Streptomyces sp ensaiadas apresentaram atividade antifúngica frente às cepas de $\mathrm{C}$. albicans testadas. 0 diâmetro do halo de inibição variou de 9 a $31 \mathrm{~mm}$ dependendo da cepa de Streptomyces sp e da cepa de C. albicans indicadora (Tabela 2). A cepa de Streptomyces sp ADU2.1 apresentou, em média, maior atividade frente às diferentes cepas de C. albicans.

\begin{tabular}{|c|c|c|c|c|c|}
\hline \multicolumn{6}{|c|}{ Diâmetro dos halos de inibição (mm) } \\
\hline Cepas de & Streptomyces sp & Streptomyces sp & Streptomyces sp & Streptomyces sp & Streptomyces sp \\
\hline Candida albicans & ADU1.3 & ADU 2.1 & TIJA2 & TIJA 12 & TILA 1 \\
\hline 1 & 25 & 30 & 23 & 14 & 23 \\
\hline 2 & 25 & 20 & 25 & 17 & 25 \\
\hline 5 & 28 & 30 & 28 & 19 & 26 \\
\hline 13 & 25 & 30 & 23 & 11 & 22 \\
\hline 22 & 25 & 20 & 21 & 9 & 20 \\
\hline 36 & 25 & 30 & 23 & 15 & 20 \\
\hline 41 & 26 & 31 & 25 & 12 & 22 \\
\hline 47 & 23 & 27 & 28 & 20 & 24 \\
\hline CBS562 & 26 & 30 & 22 & 13 & 23 \\
\hline
\end{tabular}

* Parâmetros estatísticos de análise da tabela : média, análise de variância, desvio padrão, erro padrão e coeficiente de variação: $\mathrm{x}=22,87 ; \mathrm{S}^{2}=28,47 ; \mathrm{s}=5,33 ; \mathrm{Sx}=0,84$ e CV = 23,3\%

\section{DISCUSSÃ0}

Com base no censo populacional de 2000, a população infantil brasileira na faixa etária de 0 a 10 anos é aproximadamente de 36,3 milhões de crianças ${ }^{9}$. Sabendo que a frequêencia de crianças acometidas por síndrome de Down é estimada no Brasil em 1:600 nascimentos ${ }^{18} 1920$, teríamos, portanto 60,5 mil crianças portadoras desta síndrome, equivalendo aproximadamente a $0,2 \%$ da população infantil presente em nosso País. Esta realidade humana constitui sempre um desafio aos profissionais de saúde em busca de melhor condição de vida a estes futuros cidadãos brasileiros, inclusive no que diz respeito à saúde bucal e precisamente à bacteriologia e a micologia clínica.

A síndrome de Down é decorrente da trissomia do cromossomo 21 do cariótipo humano, a qual pode ser provocada por não-disjunção, translocação ou ainda mosaicismo ${ }^{20}$. Esta cromossomopatia induz alterações bucais que afetam estruturas ósseas, como os dentes, língua gengiva e mucosa. Deste modo, a capacidade de colonização e/ou patogenicidade das cepas de Candida pode ser favorecida pela protusão da língua, respiração bucal, irritação da mucosa com fissuras linguais e nos cantos labiais, problemas respiratórios e higiene bucal deficiente ${ }^{171819}$.
Agrega-se ainda a esta situação, o comprometimento do sistema imunológico em relação as respostas celular e humoral. Linfócitos T e células killer possuem suas atividades funcionais afetadas. Niveis baixos de imunoglobulinas $\mathrm{IgG}_{2}$ e $\mathrm{IgG}_{4}$ favorecem infecções microbiológicas, além da irregularidade fisiológica da peróxido-desmutase comprometer a defesa orgânica contra microrganismos, levando cepas de Candida e Staphylococcus a serem agentes etiológicos predominantes nos processos infecciosos bucais ${ }^{19}$. 0 carreamento de leveduras de Candida na boca das crianças com síndrome de Down pode ser assim apontado como um provável fator indutor à candidíase bucal ${ }^{419}$. Em nosso estudo, a elevada detecção de isolados de C. albicans presentes na mucosa bucal de crianças portadoras desta alteração cromossômica em relação ao grupo controle (crianças sem síndrome de Down) mostrou a predisposição significativa que estas crianças apresentam a esta patologia bucal induzida por este fungo leveduriforme. Carstedt e $\mathrm{cols}^{4} \mathrm{e}$ Ribeiro e cols ${ }^{19}$ presenciaram esta mesma realidade trabalhando com prevalência de Candida na cavidade bucal de crianças e adolescentes com síndrome de Down suecos e brasileiros respectivamente. No entanto, o grupo controle (crianças sem síndrome de Down) apresentou uma ocorrência de leveduras de C. albicans presentes na boca um pouco abaixo da comumente detectada em crianças assindrômicas ${ }^{18} 1925$. 
Apesar de todo arsenal antifúngico disposto no mercado, relatos de cepas de Candida resistentes a estes fármacos são cada vez mais comuns e as recidivas infecções bucais por estas leveduras em crianças com síndrome de Down estimulam a busca de potenciais alternativas terapêuticas que venham favorecer 0 surgimento de novos medicamentos ${ }^{162325}$.

Os actinomicetos, especialmente os Streptomyces, têm sido descritos como as maiores fontes de antibióticos desde que Waksman os introduziu num programa sistemático de seleção de novos antibióticos a partir de $1940^{14}$. A abordagem para a pesquisa e descobrimento de novos antibióticos é geralmente baseada na seleção de actinomicetos que ocorrem naturalmente ${ }^{12}$. Erwealor \& Njoku-Obi5 e Lee \& Hwang ${ }^{11}$ trabalhando com 0 isolamento e seleção de Streptomyces em solos africanos e coreanos, respectivamente, isolaram diversas espécies com atividade antifúngica, demonstrando que os ensaios in vitro para a seleção de compostos antifúngicos apresentaram grande sucesso.

Resultados preliminares obtidos pelo nosso grupo de trabalho sugerem um grande potencial na descoberta de novos produtos naturais biologicamente ativos de actinomicetos isolados de diferentes solos brasileiros ${ }^{124}$. As substâncias bioativas produzidas por estes actinomicetos têm mostrado nos últimos anos uma das alternativas mais promissoras no desenvolvimento de novas drogas antimicrobianas, antitumorais e enzimáticas ${ }^{16112324}$. Ainibição de cepas de C. albicans, procedentes da cavidade bucal de crianças com síndrome de Down, pelos isolados de Streptomyces sp. testados, tem sugerido que novas condutas terapêuticas para 0 tratamento de candidíase bucal com recidivas freqüentes apresente-se viável no futuro próximo $0^{6}$.

\section{REFERÊNCIAS BIBLIOGRÁFICAS}

1. Azevedo, RCLS, Pimenta, FC, Vieira, JDG. Determinação da atividade antimicrobiana de Actinoplanes isolados do solo de cerrado goiano e efeito citotóxico do extrato etanólico bruto dos isolados. Revista de Patologia Tropical 33: 217-226, 2004.

2. Birman EG. Um breve retrospecto sobre Candida e candidoses em relação à boca. Revista Vida 8: 56-59, 1998.

3. Calderone RA, Fonzi WA. Virulence factors of C. albicans. Trends Microbiology 9: 327-335, 2001.

4. Carlstedt K, Krekmanova L, Dahllof G, Ericsson B, Braathen G, Modeer T. Oral carriage of Candida species children and adolescents with Down's syndrome. International Journal Paediatric Dentistry 6: 95-100, 1996.

5. Erwealor IA, Njoku-Obi ANU. Antimicrobial activity of a Streptomyces species isolated from Nsukka soil. World Journal Microbiology Biotechnology 6: 337-339, 1990.
6. Furlan RLA. Obtenção e estudo de mutantes com produção alterada do antibiótico retamicina sintetizado por Streptomyces olindensis. Dissertação de Mestrado, Universidade de São Paulo, São Paulo, SP, 1997.

7. Henning M, Perrone M. Prótesis odontológica en la ecología de C. albicans en cavidad bucal. Acta Odontológica Venezolana 39: 78-84, 2002.

8. Kreger-van RIJ NW: The yeast: a taxonomic study. Elsevier, Amsterdam, 1984.

9. Instituto Brasileiro de Geografia e Estatística. Censo populacional, 2000. www.ibge.gov.br. Acesso em 28.02.2004.

10. Lacaz CS, Porto E, Heins-Vacarri EM, Melo NT. Guia para identificação de fungos, actinomicetos e algas de interesse médico. Sarvier, São Paulo, 1999.

11. Lee JY, Hwang BK. Diversity of antifungal actinomycetes in various vegetative soils of Korea. Canadian Journal of Microbiology 8: 407-417, 2002.

12. Linossier A, Vargas A, Villegas R, Chimenos E. Quantitative relationship between salivary level of $\mathrm{S}$. mutans and $\mathrm{C}$. albicans in children with Down's syndrome. Journal Medicine Oral 7: 284-292, 2002.

13. Maitan VR. Isolamento e caracterização de actinomicetos endofíticos isolados de Solanum lycocarpum (lobeira). Dissertação de Mestrado. Universidade Federal de Goiás, Goiânia, G0, 1998.

14. Niroki N, Hamada T, Yamamoto T. Denture plaque - past and recent concerns. Journal of Dentistry 26: 299-304, 1998.

15. Nolan RD, Cross T. Isolation and screening of Actiniomycetes In: Cross T (ed) Actinomycetes in Biotechnology, Academic Press, London, p. 56-73, 1988.

16. Okami Y, Hotta K. Search and discovery of new antibiotic In: Goodfellow ST, Willians MM (eds) Actinomycetes in Biotechnology, Academic Press, London, p. 37-67, 1988.

17. Pardi G, Cardozo EI, Perrone M, Salazar E. Deteccion de espéceis de Candida en pacientes con estomatis sub-protésica. Acta Odontológica Venezolana 39: 62-69, 2002.

18. Pires CC, Silva FA, Netto JV. Síndrome de Down - Implicações odontológicas e possibilidades terapêuticas. Anais do II Congresso Brasileiro e I Encontro Latino-Americano sobre síndrome de Down, Brasília p. 59-62, 1997.

19. Ribeiro EL, Campos CCC, Crespo AMCC, Castro JS, Rocha FP, Alves M, Goulart MS, Cardoso C, Ferreira W, Naves PL, Soares AJ, Ribeiro SM, Pimenta FC. Detecção de C. albicans fosfolipidolíticas isoladas da saliva de crianças com síndrome de Down. Acta Médica Portuguesa 3: 171-174, 2002.

20. Roncario AM, Rodrigues AB, Elias MS. Síndrome de Down e Odontologia. Investigação 6: 70-74, 2002.

21. Sant'Ana PJP, Assad AL. 0 Contexto brasileiro para a bioprospecção. Biotecnologia: Ciência e Desenvolvimento 29:32-37, 2002.

22. Sato S. Produção de antibióticos. In: Lima UA, Aquarone E, Borzani, W, Schmidell W (eds) Biotecnologia industrial - Processos fermentativos e enzimáticos. Editora Edgard Blücher Ltda, São Paulo, p.101-124, 2001.

23. Semêdo LTAS. Atividade antimicrobiana e celulolítica de actinomicetos isolados de solos brasileiros. Dissertação de Mestrado, Universidade Federal do Rio de Janeiro, Rio de Janeiro, RJ, 1997.

24. Vieira JDG. Purificação e caracterização de uma a-amilase de Streptomyces sp. Tese de Doutorado, Universidade de São Paulo, São Paulo, SP, 1999.

25. Webb BC, Thomas CJ, Willcox MDP, Harty DWS, Knox KW: Candidaassociated denture stomatitis. Aetiology and management: A review. Part 2. Oral diseases caused by Candida species. Australian Dentistry Journal 43: 160-161, 1998. 